\title{
ASYMPTOTIC BEHAVIOR OF SOLUTIONS OF NONLINEAR FUNCTIONAL DIFFERENTIAL EQUATIONS IN BANACH SPACE
}

\author{
BY
}

JOHN R. HADDOCK

\begin{abstract}
Let $X$ be a Banach space and let $C=C([-r, 0], X)$ denote the space of continuous functions from $[-r, 0]$ to $X$. In this paper the problem of convergence in norm of solutions of the nonlinear functional differential equation $\dot{x}=F\left(t, x_{t}\right)$ is considered where $F:[0, \infty) \times C \rightarrow X$. As a special case of the main theorem, stability results are given for the equation $\dot{x}(t)=f(t, x(t))+g\left(t, x_{t}\right)$, where $-f(t, \cdot)-\alpha(t) I$ satisfies certain accretive type conditions and $g(t, \cdot)$ is Lipschitzian with Lipschitz constant $\beta(t)$ closely related to $\alpha(t)$.
\end{abstract}

1. Introduction. Let $X$ be a Banach space with norm $\|\cdot\|$ and let $C$ $=C([-r, 0], X)$ denote the space of continuous functions which map the interval $[-r, 0]$ into $X$, where $r>0$ is given. For $\phi \in C$, define $\|\phi\|_{C}$ $=\max _{-r \leqslant s \leqslant 0}\|\phi(s)\|$. If $x: I \rightarrow X$ is continuous on $I=\left[t_{0}-r, t_{0}+a\right)$ for some $a>0$, then, for each $t \in\left[t_{0}, t_{0}+a\right), x_{t} \in C$ is defined by $x_{t}(s)$ $=x(t+s),-r \leqslant s \leqslant 0$. We consider the nonlinear, nonautonomous functional differential equation

$$
\dot{x}\left(t_{0}, \phi\right)(t)=F\left(t, x_{t}\left(t_{0}, \phi\right)\right), \quad x_{t_{0}}\left(t_{0}, \phi\right)=\phi, \quad t \geqslant t_{0}
$$

where $F:[0, \infty) \times C_{F} \rightarrow X, C_{F} \subset C$. For a discussion of examples from which equations such as (1.1) arise, we refer to $\S \S 1$ and 5 of [11].

The purpose of this paper is to provide sufficient conditions for convergence in norm and convergence of solutions of equation (1.1). We find it convenient (although not necessary) to assume throughout this paper that

$$
F(t, 0)=0 \text { for all } t \geqslant 0 .
$$

The principal technique involved depends on a close examination of the right derivative with respect to $F$ of the norm $\|\cdot\|$ of $X$ defined by

Received by the editors January 26, 1976.

AMS (MOS) subject classifications (1970). Primary 34G05.

Key words and phrases. Banach space, nonlinear differential equation, accretive operator, stability. 


$$
D^{+}(t, \phi(0), \phi, F) \stackrel{\text { def }}{=} \lim _{h \rightarrow 0^{+}} \frac{1}{h}(\|\phi(0)+h F(t, \phi)\|-\|\phi(0)\|)
$$

for all $(t, \phi)$ in the domain of $F$. We note that this limit always exists (cf. [8, $\S I I .5])$. We are particularly interested in examining $D^{+}(t, \phi(0), \phi, F)$ with respect to certain subsets of $C$. One such important subset is the set $C_{0}$ defined by

$$
C_{0}=\left\{\phi \in C:\|\phi\|_{C}=\|\phi(0)\|\right\}
$$

and we rely heavily on the inequality

$$
D^{+}(t, \phi(0), \phi, F) \leqslant 0 \text { for all } \phi \in C_{0} \cap C_{F} \text { and } t \geqslant 0 .
$$

As a consequence of our main result on convergence in norm of solutions, we prove (Theorem 4.1 and Remark 4.1) stability and asymptotic stability of equation (1.1) when the right-hand side takes the more specialized form

$$
F(t, \phi)=f(t, \phi(0))+g(t, \phi),
$$

where the "ordinary" part $f$ in some sense dominates the "functional" part $g$. By applying some well-known properties of accretive (monotone) operators, we obtain, as a special case of our work, recent results of Webb [12] and Bressan and Dyson [2], where it is assumed $-f-\alpha I$ satisfies certain accretive type conditions and $g$ is Lipschitzian with Lipschitz constant closely related to $\alpha$. This will be discussed in detail in $\$ 4$.

2. Preliminary remarks and results. By a solution of $(1.1)$ on $I_{0}=\left[t_{0}, \infty\right)$, we mean a function $x\left(t_{0}, \phi\right)(\cdot)=x(\cdot)$, absolutely continuous on bounded intervals of $I_{0}$, such that $x_{t_{0}}=\phi$ and $\dot{x}(t)=F\left(t, x_{t}\right)$ a.e. on $I_{0}$. It is fundamentally important to us that the following condition is satisfied:

$$
\left\{\begin{array}{l}
\text { If } x\left(t_{0}, \phi\right)(\cdot)=x(\cdot) \text { is a solution of }(1.1) \text { on } I_{0}=\left[t_{0}, \infty\right), \text { then } \\
D^{+}(\|x(t)\|)={ }^{\operatorname{def}} \lim _{h \rightarrow 0^{+}}(\|x(t+h)-x(t)\|) / h=D^{+}\left(t, x_{t}(0), x_{t}, F\right) \\
\text { for any } t \in I_{0} \text { such that } \dot{x}(t)=F\left(t, x_{t}\right) .
\end{array}\right.
$$

RemarK 2.1. If $F$ is continuous on $\left[t_{0}, \infty\right) \times C$ and maps closed and bounded sets into bounded sets and if (1.4) holds, then (2.1) holds and, furthermore, for each $\phi \in C,(1.1)$ has a solution $x\left(t_{0}, \phi\right)(\cdot)=x(\cdot)$ on $\left[t_{0}, \infty\right)$ such that $\dot{x}(t)=F\left(t, x_{t}\right)$ for all $t \geqslant t_{0}$. This follows as a special case of the main results of Lakshmikantham, Mitchell and Mitchell [7]. If $F$ is not continuous, the problem of showing that (2.1) holds becomes slightly more difficult. This case will be briefly discussed in $\S 4$.

The following fundamental lemma and subsequent corollary are essential to our results. 
Lemma 2.1. Suppose (1.4) and (2.1) hold. If $x\left(t_{0}, \phi\right)(\cdot)$ is a solution of (1.1) on $\left[t_{0}, \infty\right)$, then $\left\|x\left(t_{0}, \phi\right)(t)\right\| \leqslant\|\phi\|_{C}$ for all $t \geqslant t_{0}$.

Proof. It follows from (1.4) and (2.1) that, for any solution $x\left(t_{0}, \phi\right)(\cdot)$ $=x(\cdot)$ of $(1.1)$ on $\left[t_{0}, \infty\right), D^{+}(\|x(t)\|) \leqslant 0$ for any $t \geqslant t_{0}$ such that $\dot{x}(t)$ $=F\left(t, x_{t}\right)$ and $\left\|x_{t}\right\|_{C}=\|x(t)\|$. Now, suppose the lemma does not hold for some $\phi$. Set $u_{0}=\|\phi\|_{C}$. Then there exists $u_{1}>u_{0}$ and $t_{1}>t_{0}$ such that $\left\|x\left(t_{0}, \phi\right)\left(t_{1}\right)\right\|=\left\|x\left(t_{1}\right)\right\|=u_{1}$. Let $t_{1}$ be the first such time that this occurs and let $\varepsilon_{0}$ be the slope of the ray with endpoint $\left(t_{0}, u_{0}\right)$ and passing through $\left(t_{1}, u_{1}\right)$. Now for each $0<\varepsilon \leqslant \varepsilon_{0}$, let $u(t, \varepsilon)$ denote the ray with slope $\varepsilon$ and with left endpoint $\left(t_{0}, u_{0}\right)$. For each such $\varepsilon$, let $t(\varepsilon)>t_{0}$ denote the first time that $\|x(t(\varepsilon))\|=u(t(\varepsilon), \varepsilon)$. Since, for each $\varepsilon, u(\cdot, \varepsilon)$ is an increasing function of $t$, we have

(i) $\left\|x_{t(\varepsilon)}\right\|_{C}=\|x(t(\varepsilon))\|$ for each $t(\varepsilon)$ and

(ii) $D^{+}(\|x(t(\varepsilon))\|) \geqslant u^{\prime}(t(\varepsilon), \varepsilon)=\varepsilon$ provided $D^{+}(\|x(t(\varepsilon))\|)$ exists.

But $D^{+}(\|x(t)\|)$ exists a.e. on $\left(t_{0}, t_{1}\right]$ and, since the set $\left\{t(\varepsilon): 0<\varepsilon \leqslant \varepsilon_{0}\right\}$ has positive measure, it follows that $D^{+}\left(\left\|x\left(t\left(\varepsilon_{1}\right)\right)\right\|\right)$ exists for some $\varepsilon_{1} \leqslant \varepsilon_{0}$. By (i) and (ii), $\left\|x_{t\left(\varepsilon_{1}\right)}\right\|_{C}=\left\|x\left(t\left(\varepsilon_{1}\right)\right)\right\|$ and $D^{+}\left(\left\|x\left(t\left(\varepsilon_{1}\right)\right)\right\|\right)=\varepsilon_{1}>0$. This contradicts properties in the first sentence in the proof and the lemma follows.

REMARK 2.2. If (1.4) is satisfied and if $F$ is continuous on $[0, \infty) \times C$ and maps closed and bounded sets into bounded sets, it follows from Remark 2.1 and Lemma 2.1 that, for any $\phi \in C$ and $t_{0} \geqslant 0, x\left(t_{0}, \phi\right)(t)$ satisfies the differential equation (1.1) on the entire interval $\left[t_{0}, \infty\right)$ and $\left\|x\left(t_{0}, \phi\right)(t)\right\|$ $\leqslant\|\phi\|_{C}$ for all $t \geqslant t_{0}$. Under these conditions on $F$, Lemma 2.1 becomes a special case of a more general comparison principle (cf. [6, Volume II, pp. 6-8]). Likewise, the proof of the general comparison result takes a somewhat simpler form since the almost everywhere condition does not arise in this case. It should be pointed out, however, that Lemma 2.1 can be extended in a straightforward manner to include the comparison principle of [6]. Since we do not need this more general result, we omit the details.

The next result follows immediately from Lemma 2.1 .

CoROLlaRY 2.1. Suppose (1.4) and (2.1) hold. If $x\left(t_{0}, \phi\right)(\cdot)$ is a solution of (1.1) on $\left[t_{0}, \infty\right)$, then $\left\|x_{t}\left(t_{0}, \phi\right)\right\|$ is a nonincreasing function of $t$ on $\left[t_{0}, \infty\right)$.

3. A general result on convergence in norm. In this section, we establish conditions with respect to $D^{+}(t, \phi(0), \phi, F)$ which guarantee the existence of the limit

$$
\lim _{t \rightarrow \infty}\left\|x\left(t_{0}, \phi\right)(t)\right\|
$$

The main result is then applied in $\S 4$ to illustrate how to obtain, under certain conditions, existence of the stronger limit 


$$
\lim _{t \rightarrow \infty} x\left(t_{0}, \phi\right)(t) \text {. }
$$

If the inequality $D^{+}\left(t, \phi_{0}(0), \phi_{0}, F\right) \leqslant 0$ is satisfied for all $t \geqslant 0$ and $\phi_{0} \in C_{0} \cap C_{F}$, it follows from Corollary 2.1 that, for any $t_{0} \geqslant 0$ and $\phi \in C$ such that $x\left(t_{0}, \phi\right)(\cdot)$ is defined on $\left[t_{0}, \infty\right)$,

$$
\lim _{t \rightarrow \infty}\left\|x_{t}\left(t_{0}, \phi\right)\right\| \text { exists }
$$

(provided, of course, (2.1) holds). By assuming the above inequality holds for a certain subset of $C$ that actually contains $C_{0}$, we establish in Theorem 3.1 that the stronger limit (3.1) exists. This limit may depend on the particular solution.

In order to emphasize that we will be dealing with sets "between" $C_{0}$ and $C$, for each $\varepsilon, \gamma$ such that $0<\gamma<\varepsilon$, we define

$$
C(\gamma, \varepsilon)=\left\{\phi \in C:\|\phi\|_{C}-\|\phi(0)\| \leqslant \gamma,\|\phi\|_{C} \leqslant 2 \varepsilon \text { and }\|\phi(0)\| \geqslant \varepsilon\right\} .
$$

Clearly, $C(\delta, \varepsilon) \subset C(\gamma, \varepsilon)$ for any $0<\delta<\gamma$. Now, let $\Lambda$ denote the set of all functions $\eta(\cdot)$ defined on $(0, \infty)$ such that $0<\eta(\varepsilon)<\varepsilon$ for each $\varepsilon>0$. Clearly, $\Lambda$ is nonempty. For each $\eta(\cdot) \in \Lambda$, define

$$
C(\eta(\cdot))=\bigcup_{\varepsilon>0} C(\eta(\varepsilon), \varepsilon) \cup\{0\}
$$

LEMMA 3.1. For each $\eta(\cdot) \in \Lambda$,

$$
C_{0} \subset C(\eta(\cdot)) \subset C
$$

and each containment is proper.

PRoof. The proof is simple. First of all, it is clear from the above definitions that $C_{0}$ is a proper subset of $C(\eta(\cdot))$. Now, let $\phi \in C$ be such that, for example, $\phi(-r)=2 \phi(0)$. Then, for any $\varepsilon, \gamma$ such that $0<\gamma<\varepsilon, \phi \notin C(\gamma, \varepsilon)$. Thus, $\phi \notin C(\eta(\cdot))$ (for any $\eta(\cdot) \in \Lambda$ ). Hence, $C(\eta(\cdot))$ is a proper subset of $C$.

THEOREM 3.1. In addition to (2.1), suppose there exists a function $\eta(\cdot) \in \Lambda$ such that

$$
D^{+}(t, \phi(0), \phi, F) \leqslant 0
$$

for all $t \geqslant 0$ and $\phi \in C(\eta(\cdot)) \cap C_{F}$. Then, for any $t_{0} \geqslant 0$ and $\phi \in C$ such that $x\left(t_{0}, \phi\right)(\cdot)$ of $(1.1)$ is defined on $\left[t_{0}, \infty\right)$,

$$
\lim _{t \rightarrow \infty}\left\|x\left(t_{0}, \phi\right)(t)\right\| \text { exists. }
$$

Proof. Suppose there exist $t_{0} \geqslant 0$ and $\phi \in C$ such that $x\left(t_{0}, \phi\right)(\cdot)$ is defined on $\left[t_{0}, \infty\right)$ and $x\left(t_{0}, \phi\right)(t)=x(t)$ does not converge in norm as $t \rightarrow \infty$. 
Since (3.3) is satisfied for all $t \geqslant 0$ and $\phi \in C_{0} \cap C_{F} \subset C(\eta(\cdot)) \cap C_{F}$, it follows from Corollary 2.1 that $\left\|x_{t}\right\|_{C}$ is a nonincreasing function of $t$. Hence, $\left\|x_{t}\right\| \rightarrow \alpha$ as $t \rightarrow \infty$ for some $\alpha \geqslant 0$. If $\alpha=0$, the proof is complete since $\|x(t)\| \leqslant \max _{t-r \leqslant s \leqslant t}\|x(s)\|=\left\|x_{t}\right\|_{C} \rightarrow 0$. So we may assume $\alpha>0$.

Let $\varepsilon>0$ be chosen such that $\varepsilon<\alpha<2 \varepsilon$. Then $0<\eta(\varepsilon)<\varepsilon$, where $\eta(\cdot) \in \Lambda$ is from the hypothesis of the theorem. (We are now ready for the crucial part of the proof.) Since $\left\|x_{t}\right\|$ is nonincreasing to $\alpha$ and $\|x(t)\|$ does not converge, a constant $\beta>0(\beta<\min \{\eta(\varepsilon) / 3, \alpha-\varepsilon\})$ and sequences $\left\{t_{n}^{\prime}\right\},\left\{t_{n}\right\}$, tending monotonically to $\infty$ as $n \rightarrow \infty$, can be chosen in such a manner that

$$
\left\|x\left(t_{n}\right)\right\| \rightarrow \alpha \text { as } n \rightarrow \infty
$$

and, for each $n$,

$$
t_{n}^{\prime}<t_{n}<t_{n+1}^{\prime} \text { and }\|\| x\left(t_{n}\right)\|-\| x\left(t_{n}^{\prime}\right) \| \mid=\beta
$$

Furthermore, $\beta,\left\{t_{n}^{\prime}\right\}$ and $\left\{t_{n}\right\}$ can be chosen such that

$$
\left|\left\|x\left(t_{n}\right)\right\|-\|x(t)\|\right| \leqslant \beta \text { for } t_{n}^{\prime} \leqslant t \leqslant t_{n} .
$$

Let $T=T(\varepsilon, \eta(\cdot))$ be chosen sufficiently large that

$$
\left\|x_{t}\right\|_{C}-\alpha<\eta(\varepsilon) / 3 \text { and }\left\|x_{t}\right\|_{C} \leqslant 2 \varepsilon
$$

for all $t \geqslant T$. Also, let $M$ be chosen such that $\left|\left\|x\left(t_{n}\right)\right\|-\alpha\right| \leqslant \eta(\varepsilon) / 3$ for all $n \geqslant M$. Finally, let $N$ be chosen sufficiently large that $t_{n}^{\prime}, t_{n} \geqslant T$ and $\|x(t)\| \geqslant \varepsilon$ for $n \geqslant N$ and $t_{n}^{\prime} \leqslant t \leqslant t_{n}$. This can be accomplished due to (3.4) and the choice of $\beta$. Therefore, for $n \geqslant \max \{M, N\}$ and $t_{n}^{\prime} \leqslant t \leqslant t_{n}$, we have

$$
\begin{aligned}
\left\|x_{t}\right\|_{C}-\left\|x_{t}(0)\right\|= & \left|\left\|x_{t}\right\|_{C}-\left\|x_{t}(0)\right\|\right| \leqslant\left|\left\|x_{t}\right\|_{C}-\alpha\right| \\
& +\left|\alpha-\left\|x\left(t_{n}\right)\right\|\right|+\left|\left\|x\left(t_{n}\right)\right\|-\|x(t)\|\right| \\
\leqslant & \eta(\varepsilon) / 3+\eta(\varepsilon) / 3+\beta \leqslant \eta(\varepsilon) .
\end{aligned}
$$

Thus, $x_{t} \in C(\eta(\varepsilon), \varepsilon) \subset C(\eta(\cdot))$ for such $t$ since $\left\|x_{t}\right\|_{C}-\left\|x_{t}(0)\right\| \leqslant \eta(\varepsilon),\left\|x_{t}\right\|_{C}$ $\leqslant 2 \varepsilon$ and $\left\|x_{t}(0)\right\| \geqslant \varepsilon$. It now follows from (3.3) that

$$
D^{+}(\|x(t)\|) \leqslant 0 \quad \text { a.e. on }\left[t_{n}^{\prime}, t_{n}\right]
$$

for each $n \geqslant \max \{M, N\}$. Hence, for $n \geqslant \max \{M, N\}$, we have

$$
\left\|x\left(t_{n}\right)\right\|-\left\|x\left(t_{n}^{\prime}\right)\right\|=\int_{t_{n}^{\prime}}^{t_{n}} D^{+}(\|x(t)\|) \leqslant 0
$$

which implies $\left\|x\left(t_{n}\right)\right\| \leqslant\left\|x\left(t_{n}^{\prime}\right)\right\|$. But $\left|\left\|x\left(t_{n}\right)\right\|-\left\|x\left(t_{n}^{\prime}\right)\right\|\right|=\beta$ for each $n$ and it follows that 


$$
\left\|x\left(t_{n}^{\prime}\right)\right\|=\left\|x\left(t_{n}\right)\right\|+\beta \geqslant \alpha+\beta / 2
$$

for sufficiently large $n$ since $\left\|x\left(t_{n}\right)\right\| \rightarrow \alpha$ as $n \rightarrow \infty$. This inequality contradicts that $\left\|x_{t}\right\|_{C} \searrow \alpha$ as $t \rightarrow \infty$ and the proof is complete.

To see that Theorem 3.1 does not, in fact imply existence of the stronger limit (3.2), we need only to consider the ordinary differential equation $(r=0)$

$$
\dot{x}_{1}=x_{2}, \quad \dot{x}_{2}=-x_{1},
$$

where, in this case, $X=R^{2}$. With euclidean norm, the conditions of Theorem 3.1 are satisfied, but no solution of (3.5) with nonzero initial condition converges.

4. Examples involving accretive type conditions. In this section, we employ our previous results to equations of the form

$$
\dot{x}\left(t_{0}, \phi\right)(t)=f\left(t, x\left(t_{0}, \phi\right)(t)\right)+\dot{g}\left(t, x_{t}\left(t_{0}, \phi\right)\right), \quad x_{t_{0}}=\phi,
$$

where $f:[0, \infty) \times X_{f} \rightarrow X, X_{f} \subset X$, and $g:[0, \infty) \times C_{g} \rightarrow X, C_{g} \subset C$. Further, we assume $f(t, 0) \equiv 0 \equiv g(t, 0)$ so the right-hand side, $F$, of equation (1.1) takes the form

$$
F(t, \phi)=f(t, \phi(0))+g(t, \phi)
$$

which was mentioned in $\S 1$. Recalling that the limit

$$
\lim _{h \rightarrow 0^{+}} \frac{1}{h}(\|x+h y\|-\|x\|)
$$

always exists for any $x, y \in X$, we have the following

THEOREM 4.1. Suppose there exist functions $\alpha, \beta, p, q:[0, \infty) \rightarrow[0, \infty)$ such that the following conditions are satisfied:

(i) $\beta(t) \leqslant \alpha(t) \gamma$ for all $t \geqslant 0$ and some $0 \leqslant \gamma<1$;

(ii) $p$ is continuous on $[0, \infty)$ with $p(0)=0$ and $p(u)>0$ if $u \neq 0$;

(iii) $q(u) \leqslant p(u)$ for all $u \geqslant 0$;

(iv) $\|g(t, \phi)\| \leqslant \beta(t) q\left(\|\phi\|_{C}\right)$ for all $(t, \phi) \in[0, \infty) \times C_{g}$; and

(v) $\lim _{h \rightarrow 0^{+}}(\|x+h f(t, x)\|-\|x\|) / h \leqslant-\alpha(t) p(\|x\|)$ for all $(t, x) \in[0, \infty)$ $\times X_{f}$.

Then condition (3.3) is satisfied for the function $F$ in (4.2) and some function $\eta(\cdot) \in \Lambda$.

Proof. For each $\varepsilon>0$, define $\Gamma(\varepsilon)=\{u: \varepsilon \leqslant u \leqslant 2 \varepsilon\}$ and let $\delta(\varepsilon)$ $=\min \{p(u): u \in \Gamma(\varepsilon)\}$ and $\sigma(\varepsilon)=\delta(\varepsilon)(1-\gamma) / 2 \gamma$. Then $\delta(\varepsilon), \sigma(\varepsilon)>0$ since $p(\cdot)$ is continuous and $p(u)>0$ if $u \neq 0$. We define $\eta(\cdot) \in \Lambda$ as follows: for each $\varepsilon>0, p(\cdot)$ is uniformly continuous on $\Gamma(\varepsilon)$, so choose $\eta(\varepsilon)>0$ such that 
$\eta(\varepsilon)<\varepsilon$ and $\left|p\left(u_{1}\right)-p\left(u_{2}\right)\right|<\sigma(\varepsilon)$ for all $u_{1}, u_{2} \in \Gamma(\varepsilon)$ with $\left|u_{1}-u_{2}\right|<\eta(\varepsilon)$. By the Axiom of Choice, we may choose each $\eta(\varepsilon)$ such that $\eta(\cdot)$ is well defined. Hence, $\eta(\cdot) \in \Lambda$. For the remainder of the proof, it suffices to show that, for each $\varepsilon>0, D^{+}(t, \phi(0), \phi, F) \leqslant 0$ for $t \geqslant 0$ and $\phi \in C(\eta(\varepsilon), \varepsilon)$ $\cap C_{F}$.

Now, for any $t \geqslant 0$ and $\phi \in C_{F}$,

$$
\begin{aligned}
D^{+}(t, \phi(0), \phi, F) & =\lim _{h \rightarrow 0^{+}} \frac{1}{h}(\|\phi(0)+h F(t, \phi)\|-\|\phi(0)\|) \\
& =\lim _{h \rightarrow 0^{+}} \frac{1}{h}(\|\phi(0)+h f(t, \phi(0))+h g(t, \phi)\|-\|\phi(0)\|) \\
& \leqslant \lim _{h \rightarrow 0^{+}} \frac{1}{h}(\|\phi(0)+h f(t, \phi(0))\|-\|\phi(0)\|)+\|g(t, \phi)\| \\
& \leqslant-\alpha(t) p(\|\phi(0)\|)+\beta(t) q(\|\phi\|) \\
& \leqslant-\alpha(t) p(\|\phi(0)\|)+\alpha(t) \gamma p(\|\phi\|) .
\end{aligned}
$$

Hence, for any $t \geqslant 0$ and $\phi \in C(\eta(\varepsilon), \varepsilon) \cap C_{F}$, it can be seen from straightforward calculations that

$$
\begin{aligned}
D^{+}(t, \phi(0), \phi, F) & \leqslant-\alpha(t) p(\|\phi(0)\|)+\alpha(t) \gamma[p(\|\phi(0)\|)+\sigma(\varepsilon)] \\
& \leqslant-\alpha(t) p(\|\phi(0)\|)[1-\gamma-\gamma \sigma(\varepsilon) / \delta(\varepsilon)] \\
& \leqslant-\alpha(t) \mu(\varepsilon) p(\|\phi(0)\|),
\end{aligned}
$$

where $\mu(\varepsilon)=1-\gamma-\gamma \sigma(\varepsilon) / \delta(\varepsilon)$. By the choice of $\sigma(\varepsilon)$, it follows that $0<\mu(\varepsilon)$ $<1$ and, therefore,

$$
D^{+}(t, \phi(0), \phi, F) \leqslant-\alpha(t) \mu(\varepsilon) \delta(\varepsilon)
$$

for $t \geqslant 0$ and $\phi \in C(\eta(\varepsilon), \varepsilon) \cap C_{F}$. Since $\varepsilon>0$ was arbitrary,

$$
D^{+}(t, \phi(0), \phi, F) \leqslant 0
$$

for any $t \geqslant 0$ and $\phi \in C(\eta(\cdot)) \cap C_{F}$. This completes the proof.

For the remainder of this paper, we indicate how to obtain and to extend recent results in [12] and [2] by using Theorem 4.1. By utilizing a general result due to Crandall and Liggett [3], Webb [12] employed nonlinear semigroup theory to prove asymptotic stability of the autonomous counterpart to equation (4.1) essentially under the conditions:

$X^{*}$, the dual space of $X$ is uniformly convex; $-f-\alpha I$ is accretive; $R(I-\lambda f)=X$ for $0<\lambda<1 / \max (0, \alpha)$ (where $R(I-\lambda f)$ is the range of the operator $I-\lambda f)$; and $g$ is Lipschitzian with Lipschitz constant $\beta$, $-\alpha+\beta<0$. 
Bressan and Dyson [2] have extended Webb's results to the nonautonomous case. In order to apply our results to the conditions of [2], we need the following brief discussion.

Let $Y$ be an arbitrary Banach space with norm $\|\cdot\|$ and let $A: Y_{A} \rightarrow Y$ be an operator on $Y_{A} \subset Y$. Further, let $L(A)$ denote the smallest number such that: for each bounded set $Q \subset Y_{A}$ such that $A(Q)$ is bounded and for each $\beta>0, \varepsilon>0$ there is a number $\delta$ such that

$$
(\|x-y+h[A x-A y]\|-\|x-y\|) / h \leqslant L(A)\|x-y\|+\varepsilon
$$

whenever $0<h \leqslant \delta$ and $x, y \in Q$ with $\|x-y\| \geqslant \beta(L(A)$ may be $\infty)$. An operator $A: Y_{A} \rightarrow Y$ is said to be accretive (monotone) if

$$
\|x-y+\lambda[A x-A x]\| \geqslant\|x-y\|
$$

for all $x, y \in Y_{A}$ and $\lambda>0$.

By assuming that, for each fixed $t,-f(t, \cdot)-\alpha(t) I$ satisfies the conditions of (4.4), we have from well-known properties of $L(\cdot)$ with respect to accretive operators that

$$
L(-(-f(t, \cdot)-\alpha(t) I))=L(f(t, \cdot))+\alpha(t) \leqslant 0 .
$$

For details, see, for instance, [9]. Hence,

$$
L(f(t, \cdot)) \leqslant-\alpha(t)
$$

for all $t \geqslant 0$. Therefore, by setting $y=0$ and recalling that $f(t, 0) \equiv 0$, it follows from the definition of $L(\cdot)$ that

$$
\lim _{h \rightarrow 0}(\|x+h f(t, x)\|-\|x\|) / h \leqslant L(f(t, \cdot))\|x\| \leqslant-\alpha(t)\|x\|
$$

for each $(t, x) \in[0, \infty) \times X_{f(t,)}$.

RemarK 4.1. If $-f(t, \cdot)-\alpha(t) I$ satisfies the conditions of (4.4) and if $\|g(t, \phi)\| \leqslant \beta(t)\|\phi\|_{C}$ for all $(t, \phi) \in[0, \infty) \times C_{g(t,)}$, where $\beta(t) \leqslant \alpha(t) \gamma, 0$ $\leqslant \gamma<1$, then it follows from (4.9) that the conditions of Theorem 4.1 are satisfied for $p(u)=u=q(u), u \geqslant 0$. If, in addition, $\int^{\infty} \alpha(t) d t=\infty$, standard arguments as in [1] can be applied to show that solutions defined for all future $t$ must tend to zero. To obtain asymptotic stability of (4.1), then, requires establishing appropriate existence results. Note that under these conditions on $f$ and $g$ with $\gamma \leqslant 1$, (uniform) stability of (4.1) can be concluded directly from Corollary 2.1.

REMARK 4.2. As was mentioned above, existence results need to be established along with the stability results discussed in Remark 4.1. This can be done in one fashion via nonlinear semigroup theory, as in [12], [2] or [4], by 
applying fundamental results of Crandall and Liggett [3] and Flaschka and Leitman [5]. (See [10] for an extension of some results in [5].) For this case, we have not improved the stability results of [12] and [2], but, rather, we have approached the problem from a different and hopefully somewhat simpler viewpoint. On the other hand, if $f$ and $g$ are defined and continuous on $[0, \infty) \times X$ and $[0, \infty) \times C$, respectively, and map closed and bounded sets of their respective domains into bounded sets of $X$, then the situation becomes quite different. In fact, for this case, we actually improve the results of [12] and [2]. Existence is no longer a difficulty since, from [7], the conditions of Lemma 2.1, hence Theorem 4.1, are enough to guarantee existence of a solution $x\left(t_{0}, \phi\right)(\cdot)$ on $\left[t_{0}, \infty\right)$ for each initial condition $x_{t_{0}}=\phi$. (Note that $(2.1)$ automatically holds with $f$ and $g$ continuous.) Hence, we can apply Corollary 2.1 and Theorem 4.1 to obtain stability and asymptotic stability whenever $-f(t, \cdot)-\alpha(t) I$ is not necessarily accretive or $g(t, \cdot)$ is not necessarily Lipschitzian.

As a final comment, we note that, since our main result (Theorem 3.1) was given in a general setting, it offers more flexibility in applications than results designed specifically for equation (4.1). Also, it should be pointed out that Theorem 3.1 is new even for the more specialized but very important finite dimensional case $X=R^{n}$. This result, then, can be used to generalize and extend previous results for this special case. See, for instance, [1] and [13].

AcKnowledgement. The author is deeply indebted to Glenn F. Webb for several valuable discussions and suggestions concerning this paper. The author is also very grateful to Robert $\mathrm{H}$. Martin, Jr. for having made available an advanced copy of the manuscript listed in [8].

\section{REFERENCES}

1. S. R. Bernfeld and J. R. Haddock, Liapunov-Razumikhin functions and convergence of solutions of scalar functional differential equations.

2. R. V. Bressan and J. Dyson, Functional differential equations and nonlinear evolution operators, Edinburgh J. Math. (to appear).

3. M. G. Crandall and T. M. Liggett, Generation of semi-groups of nonlinear transformations on general Banach spaces, Amer. J. Math. 93 (1971), 265-298. MR 44 \# 4563.

4. W. E. Fitzgibbon, Stability for abstract nonlinear Volterra equations involving finite delay.

5. H. Flaschka and M. J. Leitman, On semigroups of nonlinear operators and the solution of the functional differential equation $\dot{x}(t)=F\left(x_{t}\right)$, J. Math. Anal. Appl. 49 (1975), 649-658.

6. V. Lakshmikantham and S. Leela, Differential and integral inequalities, Vol. II, Academic Press, New York, 1969.

7. V. Lakshmikantham, A. R. Mitchell and R. W. Mitchell, On the existence of solutions of differential equations of retarded type in a Banach space.

8. R. H. Martin, Jr., Nonlinear operators and differential equations in Banach spaces, Interscience, New York, 1976.

9. Japan 22 (1970), 411-429. MR 45 \#7519. 
10. A. T. Plant, Nonlinear semigroups of translations in Banach space generated by functional differential equations.

11. C. C. Travis and G. F. Webb, Existence and stability for partial functional differential equations, Trans. Amer. Math. Soc. 200 (1974), 395-418.

12. G. F. Webb, Asymptotic stability for abstract nonlinear functional differential equations, Proc. Amer. Math. Soc. 54 (1976), 225-230.

13. E. Winston, Asymptotic stability for ordinary differential equations with delayed perturbations, SIAM J. Math. Anal. 5 (1974), 303-308. MR 50 \#7750.

Department of Mathematical Sciences, Memphis State University, Memphis, Tennessee 38152 